\title{
RIGOROUS DERIVATION OF THE X-Z SEMIGEOSTROPHIC EQUATIONS *
}

\author{
YANN BRENIER ${ }^{\dagger}$ AND MIKE CULLEN $\ddagger$
}

Abstract. We prove that smooth solutions of the semigeostrophic equations in the incompressible $x-z$ setting can be derived from the Navier-Stokes equations with the Boussinesq approximation.

Key words. Semigeostrophic, Navier-Stokes equations, asymptotic analysis.

AMS subject classifications. 86A10, 35Q86, 76B03, 76B60, 86A05.

\section{Introduction} $(\mathrm{NSB})$ :

We consider the Navier-Stokes equations with the Boussinesq approximation

$$
\begin{gathered}
\epsilon\left(\partial_{t} v+(v \cdot \nabla) v\right)+\alpha K v+\nabla p=y, \nabla \cdot v=0, \\
\partial_{t} y+(v \cdot \nabla) y=G(x, y),
\end{gathered}
$$

where $x \in D, D$ being a smooth bounded domain in $R^{d}(d=2,3), v=v(t, x) \in R^{d}$ is the velocity field, $p=p(t, x)$ is the pressure field, $y=y(t, x) \in R^{d}$ is a vector-valued forcing term, $G(x, y)$ is a given smooth vector-valued source term $D \times R^{d} \rightarrow R^{d}, \epsilon, \alpha>0$ are scaling factors and $K$ is the linear dissipative operator $K v=-\Delta v$. We assume that the fluid sticks to the boundary: $v=0$ along $\partial D$.

We now consider the formal limit of these equations obtained by dropping the inertia term and the dissipative term (i.e. setting $\varepsilon=\alpha=0$ ) in the NSB equations,

$$
\begin{gathered}
\nabla p=y, \nabla \cdot v=0, v \| \partial D \\
\partial_{t} y+(v \cdot \nabla) y=G(x, y) .
\end{gathered}
$$

We are going to show that these equations can be justified under a strong uniform convexity assumption on the pressure field $p$. The situation of interest in this paper is the case when $d=2$ and the source term

$$
G(x, y)=\left(x_{2}, y_{1}-x_{1}\right) .
$$

Then (1.3),(1.4) are the semigeostrophic Eady model equations in the special incompressible " $x-z$ " situation. By $x-z$, we mean that $D$ is part of a vertical section, the second coordinate $x_{2}$ of each point $x=\left(x_{1}, x_{2}\right) \in D$ being the vertical one. The source term in (1.5) represents the effect of the missing third dimension. In this identification, $y$ represents the effects of rotation and stratification, and the relation $\nabla p=y$ in (1.3) expresses geostrophic and hydrostatic balance.

\footnotetext{
*Received: May 13, 2009; accepted: May 25, 2009. Communicated by Shi Jin.

${ }^{\dagger}$ CNRS, FR 2800, Université de Nice, Mathématiques, Parc Valrose FR-06108 Nice, France (brenier@unice.fr).

$\ddagger$ Met Office, Exeter, UK (mike.cullen@metoffice.gov.uk). M. Cullen’s contribution is Crown Copyright.
} 
The semigeostrophic model was considered by Hoskins [11] to model front formation in atmospheric sciences. The Eady model is defined in chapter 6 of [10], and models a quasi-periodic evolution in which fronts form and decay. There has been a lot of interest in these equations (see for instance $[9,1,7,6,10]$ ), due to their beautiful geometric structure and their deep links with the Monge-Ampère equation and optimal transport theory $[5,2,3,13]$. The rigorous derivation of the full 3 dimensional $\mathrm{SG}$ equations is still a challenging problem. The present short note is just the first step toward this goal.

2. Motivation for a convexity assumption In their study of the SG equations, Cullen and Purser have introduced a convexity assumption on the pressure field $p$, based on a combination of physical and mathematical arguments. Convexity is also natural in the case of the general equations (1.3)-(1.4), independently of the choice of the source term $G$, for the following reasons. At first glance, these equations look strange since there is no evolution equation for $v$. However, $y$ is constrained to be a gradient. Therefore, $v$ can be seen as a kind of Lagrange multiplier for this constraint. (Vaguely speaking, due to the presence of a source term, in order to stay a gradient, the field $y$ needs to be continuously rearranged in a volume-preserving fashion under the action of a time-dependent divergence-free vector field $v$.) As a matter of fact, it is (formally) very easy to get an equation for $v$, once $y=\nabla p$ is known. To do that, let us start with the 2 dimensional case and write

$$
y(t, x)=\left(\partial_{1} p, \partial_{2} p\right)\left(t, x_{1}, x_{2}\right), v(t, x)=\left(-\partial_{2} \psi, \partial_{1} \psi\right)\left(t, x_{1}, x_{2}\right)
$$

(at least locally), where $\psi$ is a "stream-function". Then, let us "curl" equation (1.4) and obtain

$$
-\partial_{11}^{2} p \partial_{22}^{2} \psi+2 \partial_{12}^{2} p \partial_{12}^{2} \psi-\partial_{22}^{2} p \partial_{11}^{2} \psi=\partial_{1}\left(G_{2}(x, \nabla p)\right)-\partial_{2}\left(G_{1}(x, \nabla p)\right) .
$$

This is a linear second order elliptic equation in $\psi$, whenever $p$ is a given strictly uniformly convex (or concave) function of $x$, i.e., when $D_{x}^{2} p>0$, in the sense of symmetric matrices, (or $<0)$. In three space dimensions, we get some "magnetostatic" version of equation (2.1). Indeed, since $v$ is divergence-free, we can (at least locally) write $v=\nabla \times A$ for some "potential vector" $A=A(t, x) \in R^{3}$, that we may assume to be itself divergence-free. Then, by curling equation (1.4), we get a linear system for $A$ when $p$ is convex, namely:

$$
\nabla \times(M(t, x) \nabla \times A)=\nabla \times(G(x, \nabla p)) .
$$

This system is elliptic whenever the symmetric matrix $M=D_{x}^{2} p(t, x)$ is uniformly positive and bounded, which means that $p$ is convex in a strong sense. In higher dimensions, $v$ should be viewed as a $d-1$ form and $p$ as a zero form. The divergence free condition (locally) means that $v=d A$, where $A$ is a $d-2$ form. Then, again taking the curl of equation (1.4), we get the multidimensional generalization of system (2.1): $d(M(t, x) * d A)=d\left(G(x, d p)\right.$ (where * denotes Hodge duality and $M=D^{2} p$ ) which, again, is an elliptic system in $A$ when $D_{x}^{2} p$ is uniformly bounded and positive.

Thus we see that requiring $p$ to be convex is a natural solvability condition for equations (1.3)-(1.4).

\section{Rigorous derivation from the Navier-Stokes equations}

The generalized Cullen-Purser convexity condition plays a crucial role in the rigorous derivation of equations (1.3)-(1.4) from the NSB equations. 
TheOrem 3.1. Let $D$ be a smooth bounded convex domain. Assume $G$ to be smooth with bounded derivatives up to second order. Let $\left(y^{\varepsilon}, v^{\varepsilon}, p^{\varepsilon}\right)$ be a Leray-type solution to the NSB equations (1.1),(1.2), where $K=-\Delta$, with $\alpha=O(\varepsilon)$. Let $(y=\nabla p, v)$ be a smooth solution to equations (1.3),(1.4) on a given finite time interval $[0, T]$. We assume $p(t, x)$ to have a smooth convex extension for all $x \in R^{d}$ so that its Legendre transform

$$
p^{*}(t, y)=\sup _{x \in R^{d}} x \cdot y-p(t, x)
$$

is also smooth for $y \in R^{d}$ with Hessian $D_{y}^{2} p^{*}(t, y)$ bounded away from zero and $+\infty$.

Then, the $L^{2}$ distance between $y^{\varepsilon}$ and $y$ stays uniformly of order $\sqrt{\varepsilon}$ as $\varepsilon$ goes to zero, uniformly in $t \in[0, T]$, provided it does at $t=0$ and the initial velocity $v^{\varepsilon}(t=0, x)$ stays uniformly bounded in $L^{2}$.

Notice that the theorem is meaningful, since the local existence of smooth solutions has been proven by Loeper [12] (in the SG case) at least for periodic boundary conditions, provided that that $y(0, x)-x$ is not too large in some appropriate sense.

Proof. For the convergence, we use a relative entropy trick quite similar to the one used by the author for the hydrostatic limit of the 2D Euler equations in a thin domain [4]. We introduce the so-called Bregman function (or relative entropy) attached to $p^{*}$

$$
\eta_{p^{*}}\left(t, z, z^{\prime}\right)=p^{*}\left(t, z^{\prime}\right)-p^{*}(t, z)-\left(\nabla p^{*}\right)(t, z) \cdot\left(z^{\prime}-z\right) \sim\left|z^{\prime}-z\right|^{2}
$$

and the related functional

$$
e(t)=\int_{D}\left(\epsilon \frac{\left|v^{\varepsilon}(t, x)-v(t, x)\right|^{2}}{2}+\eta_{p^{*}}\left(t, y(t, x), y^{\varepsilon}(t, x)\right)\right) d x .
$$

Given a weak solution $\left(y^{\varepsilon}, v^{\varepsilon}\right)$ to the NSB equations (1.1),(1.2) and a solution $y$ of (1.3),(1.4), we want to get an estimate of the form:

$$
\frac{d}{d t}(e(t)+O(\epsilon)) \leq(e(t)+O(\epsilon)) c
$$

where $c$ depends only on the limit solution $(y, v)$ on a fixed finite time interval $[0, T]$ on which $(y, v)$ is smooth. From this estimate (3.4), we immediately get that $y-y^{\varepsilon}$ is of order $O(\sqrt{\epsilon})$ in $L^{\infty}\left([0, T], L^{2}(D)\right)$. So, we are left with proving (3.4). To save time, we do calculations just as if the Leray solutions were smooth solutions. Let us compute

$$
\begin{gathered}
I=I_{1}+I_{2}+I_{3}+I_{4}, \\
I_{1}=\frac{d}{d t} \int_{D} p^{*}\left(t, y^{\varepsilon}(t, x)\right) d x \\
I_{2}=-\frac{d}{d t} \int_{D} p^{*}(t, y(t, x)) d x \\
I_{3}=-\frac{d}{d t} \int_{D}\left(\nabla p^{*}(t, y(t, x))\right) \cdot y^{\varepsilon}(t, x) d x
\end{gathered}
$$




$$
I_{4}=\frac{d}{d t} \int_{D}\left(\nabla p^{*}(t, y(t, x))\right) \cdot y(t, x) d x
$$

We first obtain

$$
I_{1}=\int_{D}\left[\left(\partial_{t} p^{*}\right)\left(t, y^{\varepsilon}(t, x)\right)+\left(\nabla p^{*}\right)\left(t, y^{\varepsilon}(t, x)\right) \cdot G\left(x, y^{\varepsilon}(t, x)\right)\right] d x,
$$

(using that $v^{\varepsilon}$ is divergence free and parallel to $\partial D$ ). Similarly,

$$
I_{2}=-\int_{D}\left[\left(\partial_{t} p^{*}\right)(t, y(t, x))+\left(\nabla p^{*}\right)(t, y(t, x)) \cdot G(x, y(t, x))\right] d x .
$$

Next,

$$
\begin{gathered}
I_{3}=-\int_{D}\left[\left(\partial_{t} \nabla p^{*}\right)(t, y(t, x)) \cdot y^{\varepsilon}(t, x)+\left(D_{y}^{2} p^{*}\right)(t, y(t, x))\left(\partial_{t} y(t, x), y^{\varepsilon}(t, x)\right)\right] d x \\
-\int_{D}\left(\nabla p^{*}\right)(t, y(t, x)) \cdot G\left(x, y^{\varepsilon}(t, x)\right) d x+I_{5},
\end{gathered}
$$

where

$$
\begin{aligned}
I_{5} & =\int_{D} x \cdot\left(v^{\varepsilon}(t, x) \cdot \nabla\right) y^{\varepsilon}(t, x) d x \\
& =-\int_{D} v^{\varepsilon}(t, x) \cdot y^{\varepsilon}(t, x) d x,
\end{aligned}
$$

(where we have used, for the two last lines, that $\left(\nabla p^{*}\right)(t, y(t, x))=x$, which follows from Legendre duality). Since $v^{\varepsilon}$ solves the NSB equations, we find

$$
\begin{aligned}
I_{5}= & -\int_{D}\left[\varepsilon\left(\partial_{t}+v^{\varepsilon} \cdot \nabla\right) v^{\varepsilon}+\nabla p^{\varepsilon}+\alpha K v^{\varepsilon}\right] \cdot v^{\varepsilon} d x \\
& =-\frac{\varepsilon d}{2 d t} \int_{D}\left|v^{\varepsilon}\right|^{2} d x-\int v^{\varepsilon} \cdot \alpha K v^{\varepsilon} d x .
\end{aligned}
$$

Similarly

$$
\begin{gathered}
I_{4}=\int_{D}\left[\left(\partial_{t} \nabla p^{*}\right)(t, y(t, x)) \cdot y(t, x)+\left(D_{y}^{2} p^{*}\right)(t, y(t, x))\left(\partial_{t} y(t, x), y(t, x)\right)\right] d x+ \\
+\int_{D} \nabla p^{*}(t, y(t, x)) \cdot G(x, y(t, x)) d x .
\end{gathered}
$$

Collecting all terms, we obtain

$$
I=I_{5}+I_{6}+I_{7}+I_{8}+I_{9},
$$

where

$$
I_{6}=\int_{D} \eta_{\partial_{t} p^{*}}\left(t, y(t, x), y^{\varepsilon}(t, x)\right) d x
$$


(which involves the Bregman functional associated with $\partial_{t} p^{*}$ and therefore is bounded by $e(t) c$ where $c$ is a constant depending only on the limit solutions $y=\nabla p$ ),

$$
\begin{gathered}
I_{7}=-\int_{D}\left[\left(\nabla p^{*}\right)(t, y)-\left(\nabla p^{*}\right)\left(t, y^{\varepsilon}\right)\right] \cdot G\left(x, y^{\varepsilon}\right) d x, \\
I_{8}=\int\left(D_{y}^{2} p^{*}\right)(t, y)\left(G(x, y), y-y^{\varepsilon}\right) d x, \\
I_{9}=\int\left(D_{y}^{2} p^{*}\right)(t, y)\left(\partial_{t} y-G(x, y), y-y^{\varepsilon}\right) d x, \\
=\int\left(D_{y}^{2} p^{*}\right)(t, y)\left((v \cdot \nabla) y, y^{\varepsilon}-y\right) d x .
\end{gathered}
$$

We easily see that

$$
\begin{gathered}
I_{7}+I_{8}=\int_{D} \eta_{\nabla p^{*}}\left(t, y(t, x), y^{\varepsilon}(t, x)\right) \cdot G(x, y) d x \\
+\int_{D}\left[\left(\nabla p^{*}\right)(t, y)-\left(\nabla p^{*}\right)\left(t, y^{\varepsilon}\right)\right] \cdot\left(G(x, y)-G\left(x, y^{\varepsilon}\right)\right) d x,
\end{gathered}
$$

(which is again bounded by $e(t) c$ where $c$ is a constant depending only on the limit solutions $y=\nabla p$ ). Let us finally consider the most delicate term $I_{9}$. We can write $I_{9}$ in index notation as

$$
\begin{aligned}
& I_{9}=\int \sum_{i j k} \partial_{i j}^{2} p^{*}(t, y) v_{k} \partial_{k} y_{i}\left(y^{\varepsilon}-y\right)_{j}, \\
& =\int \sum_{i j k} \delta_{j k} v_{k}\left(y^{\varepsilon}-y\right)_{j}=\int v \cdot\left(y^{\varepsilon}-y\right),
\end{aligned}
$$

(indeed, $p^{*}$ is the Legendre transform of $p$ and $y=\nabla p$, thus $D^{2} p^{*}(y) D y=$ $\left.D^{2} p^{*}(\nabla p) D^{2} p=I d\right)$

$$
=\int v \cdot y^{\varepsilon},
$$

(since $y$ is a gradient and $v$ is divergence free and parallel to $\partial D$ )

$$
=\int v \cdot\left(\varepsilon\left(\partial_{t}+v^{\varepsilon} \cdot \nabla\right) v^{\varepsilon}+\alpha K v^{\varepsilon}\right),
$$

(using the NSB equations)

$$
=J_{1}+J_{2},
$$

where

$$
J_{1}=\frac{d}{d t} \varepsilon \int v^{\varepsilon} \cdot v
$$


and

$$
\left|J_{2}\right| \leq \varepsilon\left(\int\left|v^{\varepsilon}\right|^{2}+1\right) c \leq \varepsilon\left(\int\left|v^{\varepsilon}-v\right|^{2}+1\right) c \leq(e(t)+\varepsilon) c,
$$

where $c$ are constants only depending on the limit solution $v$. Thus, again collecting all terms, and using that

$$
I_{5}=-\frac{\varepsilon d}{2 d t} \int_{D}\left|v^{\varepsilon}\right|^{2} d x-\alpha \int v^{\varepsilon} \cdot K v^{\varepsilon} d x
$$

we have obtained

$$
I+\frac{d}{d t}\left(\frac{\varepsilon}{2} \int\left|v^{\varepsilon}-v\right|^{2}+O(\varepsilon)\right)+\alpha \int v^{\varepsilon} \cdot K v^{\varepsilon} d x \leq(e(t)+O(\varepsilon)) c,
$$

which leads to the desired inequality (3.4) and completes the proof.

Acknowledgments Yann Brenier acknowledges the support of ANR contract OTARIE ANR-07-BLAN-0235. Part of his research was done during his stay at IPAM, UCLA, program "Optimal Transport" (March 10-June 13, 2008).

\section{REFERENCES}

[1] J.-D. Benamou, Y. Brenier, Weak existence for the semigeostrophic equations formulated as a coupled Monge-Ampere/transport problem, SIAM J. Appl. Math., 58, 1450-1461, 1998.

[2] Y. Brenier, Décomposition polaire et réarrangement monotone des champs de vecteurs, C. R. Acad. Sci. Paris I Math., 305, 805-808, 1987.

[3] Y. Brenier, Polar factorization and monotone rearrangement of vector-valued functions, Comm. Pure Appl. Math., 44, 375-417, 1991.

[4] Y. Brenier, Remarks on the derivation of the hydrostatic Euler equations, Bull. Sci. Math., 127, 585-595, 2003

[5] A. Caffarelli, M. Milman (editors), Monge Ampère Equation: Applications to Geometry and Optimization, Contemporary Mathematics, 226, AMS 1999.

[6] M. Cullen, M. Feldman, Lagrangian solutions of semigeostrophic equations in physical space, SIAM J. Math. Anal., 37, 1371-1395, 2006.

[7] M. Cullen, W. Gangbo, A variational approach for the 2-D semi-geostrophic shallow water equations, Arch. Rat. Mech. Anal., 156, 241-273, 2006.

[8] M. Cullen, R. Purser, An extended Lagrangian theory of semigeostrophic frontogenesis, J. Atmospheric Sci., 41, 1477-1497, 1984.

[9] M. Cullen, J. Norbury, R. Purser, Generalised Lagrangian solutions for atmospheric and oceanic flows, SIAM J. Appl. Math., 51, 20-31, 1991.

[10] M. Cullen, A Mathematical Theory of Large-scale Atmosphere/Ocean Flow, Imperial College Press, London, 2006.

[11] B. Hoskins, The Geostrophic Momentum Approximation and the Semi-Geostrophic Equations, J. Atmospheric Sci., 32, 233-242, 1975.

[12] G. Loeper, A fully nonlinear version of the incompressible Euler equations: the semigeostrophic system, SIAM J. Math. Anal., 38, 795-823, 2006.

[13] C. Villani, Topics in Optimal Transportation,, American Mathematical Society, Providence, 2003. 